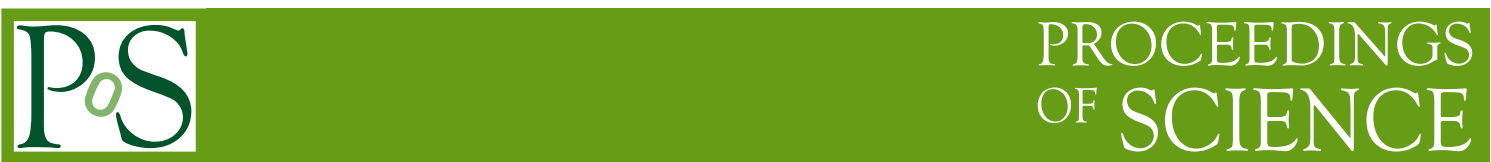

\title{
Effective Action for Low-Energy Quantum Field Fluctuations
}

\section{Raffaele Millo*}

Università degli Studi di Trento and I.N.F.N. Via Sommarive 14, Povo (Trento), Italy.

\section{Pietro Faccioli}

Università degli Studi di Trento and I.N.F.N. Via Sommarive 14, Povo (Trento), Italy.

\begin{abstract}
We present a novel approach to obtain the full non-perturbative instanton-antiinstanton interaction, using Lattice simulations. The method we describe can be applied to any family of vacuum field configurations in any Quantum Field Theory and has been tested on the 1-Dimensional double well potential. Here are discussed the results for the full non-perturbative instantonantiinstanton effective interaction in the high barrier case (where the pseudoparticles can be treated as almost non-interacting) and low barrier case.
\end{abstract}

International Workshop on QCD Green's Functions, Confinement, and Phenomenology - QCD-TNT09 September 07 - 112009

ECT Trento, Italy

* Speaker. 


\section{Introduction}

Lattice QCD allows to compute a large number of hadronic matrix elements. On the other hand, such calculations do not reveal what are the vacuum field configurations which dominate specific matrix elements. In principle, models based on a priori selection of a family relevant vacuum fields - e.g. instanton, monopoles, vortices - allow to investigate the role played by such fluctuations in different processes. For example, this way it has been possible to show that instantons play an important role in the chiral dynamics of light quarks[1,2], but do not lead to confinement of heavy quarks.

The main problem of instanton approaches is that the semiclassical approximation leading to the dilute instanton gas picture is intrinsically inconsistent in QCD. This flaw has been euristically circumvented by describing the QCD vacuum as an interacting instanton liquid, rather than a dilute gas [3]. However, this way, new problems emerge. In fact, one needs to derive the interaction between pseudo-particles including quantum corrections. On the other hand, it is not clear how to consistently generalize the instanton picture to include additional purely quantum degrees of freedom, which are responsible for confinement, without spoiling the semiclassical arguments.

In this talk we address these problems. We present a recently developed formalism which allows to consistently express the partition functions in terms of an arbitrary family of vacuum fields (not necessarily instantons) and compute the corresponding effective interaction. If the family of vacuum fields is parametrized by a set of collective coordinates, the infinite-dimensional Euclidean path integral is reduced to the canonical partition function of a finite system. Applications to QCD are currently under investigation. In this talk we test the method determining the full non-perturbative instanton-antiinstanton for a simple toy model: the quantum mechanical system described by a particle moving in a one-dimensional double well potential.

\section{Effective Interaction and Microscopic Dynamics}

Before describing the method and its applications, we briefly illustrate how the multi-instanton effective interaction can be used to gain information on microscopic dynamics of correlation functions. These considerations apply to any Quantum Field Theory but, in order to simplify the discussion, in this section we will consider the QCD case.

We start by expressing the path integral in terms of instantons coordinates $\gamma_{l}$, while all other field configurations are integrated out

$$
\begin{aligned}
Z_{Y M} & =\int \mathscr{D} A_{\mu} \exp \left\{-\frac{1}{4} G_{\mu \nu}^{a} G_{\mu \nu}^{a}\right\} \\
& \equiv \int d \gamma_{1} \ldots \int d \gamma_{k} \exp \left\{-F\left(\gamma_{1}, \ldots, \gamma_{k}\right)\right\} .
\end{aligned}
$$

where $F(\gamma)$ is the multi-instanton effective interaction. Given a matrix element

$$
\left\langle\hat{O}\left[A_{\mu}\right]\right\rangle=\frac{\int \mathscr{D} A_{\mu} O\left[A_{\mu}\right] e^{-S_{Y M}\left[A_{\mu}\right]}}{\int \mathscr{D} A_{\mu} e^{-S_{Y M}\left[A_{\mu}\right]}} .
$$

- for example the Gluon condensate, or the Topological Susceptibility-, we can perform parameter free approximate calculations using $F(\gamma)$ itself. In fact, if multi-instantons configurations 
$\tilde{A}_{\mu}(\gamma)$ are the relevant degrees of freedom driving the dynamics of the operator $O\left[A_{\mu}\right]$, this can be approximated to $O\left[A_{\mu}\right] \simeq O\left[\tilde{A}_{\mu}(\gamma)\right]$ and from Eq.(2.2) we obtain

$$
\left\langle\hat{O}\left[A_{\mu}\right]\right\rangle \simeq \frac{\int \prod_{l=1}^{k} d \gamma_{l} O\left[\tilde{A}\left(\gamma_{1}, \ldots, \gamma_{k}\right)\right] e^{-F\left(\gamma_{1}, \ldots, \gamma_{k}\right)}}{\int \prod_{l=1}^{k} d \gamma_{l} e^{-F\left(\gamma_{1}, \ldots, \gamma_{k}\right)}} .
$$

By comparing the value of the finite dimensional integral in Eq.(2.4) — which can be performed numerically - with the correct value of the matrix element given by lattice calculations or experiments, we can determine whether instantons — or other gauge field configurations - are the relevant set of degrees of freedom for the non-perturbative dynamics of the matrix element $\left\langle\hat{O}\left(A_{\mu}\right)\right\rangle$ and hence obtain qualitative informations on the role of instantons in its microscopic dynamics.

\section{From Lattice Simulations to Effective Interaction}

In this section we explain how to define and compute the effective potential for a family of vacuum configuration $\tilde{x}(t ; \gamma)$ parametrized by a set of collective coordinates $\gamma$ : in the specific case of QCD, $\gamma$ can be thought to represent instantons positions, sizes and color orientations, and possibly the collective coordinates of other types of field configurations. In order to define the effective interaction $F(\gamma)$, we have to introduce the integral over the collective coordinates in the path integral

$$
Z=\int \mathscr{D} x(t) e^{-S[x(t)]} .
$$

we do so by decomposing every field $x(t)$, in two orthogonal components

$$
x(t)=\tilde{x}(t ; \gamma)+y(t),
$$

the first component $-\tilde{x}(t ; \gamma)$ - belongs to the manifold $\mathscr{M}$ parametrized by the curvilinear coordinates $\gamma$; the second component $-y(t)$, which we call "fluctuation field" - is defined as the field orthogonal to the tangent space of the manifold $\mathscr{M}$, in the point $\bar{\gamma}$

$$
\begin{aligned}
\int \mathrm{d} t y(t) g_{\gamma_{i}}(t ; \bar{\gamma})= & \left(y \cdot g_{\gamma_{i}}(\bar{\gamma})\right)=0 ; \quad \forall i=1, \ldots, k \\
& g_{\gamma_{i}}(t ; \gamma)=\partial_{\gamma_{i}} \tilde{x}(t ; \gamma),
\end{aligned}
$$

where $\left\{g_{\gamma_{i}}(t ; \bar{\gamma})\right\}$ is an orthogonal basis of the tangent space, see Fig.(1).

The separation of the "fluctuation fields" $y(t)$ from the family of configurations $\tilde{x}(t ; \gamma)$ enables us to rewrite the path integral as

$$
\begin{aligned}
Z & =\int \prod_{l=1}^{k} d \gamma_{l} \int \mathscr{D} y\left(\prod_{i} \delta^{(k)}\left(y \cdot g_{\gamma_{i}}(\bar{\gamma})\right)\left|\operatorname{det}_{i j}\left(g_{\gamma_{i}}(\bar{\gamma}) \cdot g_{\gamma_{j}}(\gamma)\right)\right| e^{-S[\tilde{x}(t ; \gamma)+y(t)]},\right. \\
& \equiv \int \prod_{l=1}^{k} d \gamma_{l} \exp \{-F(\gamma)\}
\end{aligned}
$$

where $F(\gamma)$ is the effective interaction of the vacuum fields $\tilde{x}(t ; \gamma)$, and is defined as

$$
F(\gamma)=-\left\{\log \int \mathscr{D} y\left(\prod_{i} \delta^{(k)}\left(y \cdot g_{\gamma_{i}}(\bar{\gamma})\right)\left|\operatorname{det}_{i j}\left(g_{\gamma_{i}}(\bar{\gamma}) \cdot g_{\gamma_{j}}(\gamma)\right)\right| e^{-S[\tilde{x}(t ; \gamma)+y(t)]}\right\} .\right.
$$




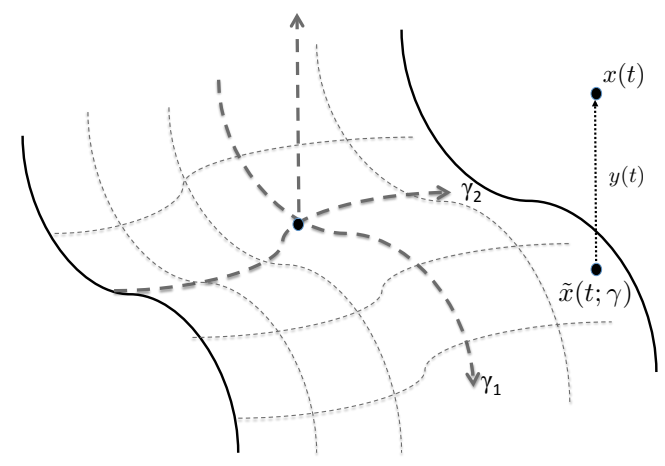

Figure 1: Pictorical representation of the projection of the field $x(t)$ onto a tangent space of the vacuum field manifold $\mathscr{M} . x(t)$ is represented by a point in this picture. The constraints (3.3) imply that the fluctuation field $y(t)$ is perpendicular to the plane tangent to the manifold in the point of the curvilinear abscissas $\gamma=\bar{\gamma}$.

We notice that the partition function (3.6) is independent on the choice of the point $\bar{\gamma}$ by contruction; on the other hand the effective interaction $F(\gamma)$ and vacuum expectation values of operators (2.4) may in principle depend on such a parameter. However, it can be shown that ${ }^{1}$ such a dependence is generated only by the projection of paths which contain very large fluctuations $y(t)$. Hence, if the vacuum fields $\tilde{x}(t ; \gamma)$ dominate the vacuum expectation values of operators $\hat{O}(t)$, this will be independent on the choice of $\bar{\gamma}$. This condition can be verified by comparing the results obtained projecting onto different points of the manifold.

We now show how to evaluate the effective interaction $F(\gamma)$. We are going to use lattice simulations, similarly to what is done in statistical physics for calculating the free energy of the reaction coordinates of a given system. We generate a set of statistical representative lattice configurations and we reconstruct numerically the profile of the effective interaction by counting the occurences of the set of collective coordinates. This is done using a projection technique which follows three steps:

1) Every configuration $x_{1}(t), \ldots, x_{N}(t)$ generated with lattice simulations is projected onto the tangent space of the vacuum manifold $\mathscr{M}$.

$$
\Psi_{i}\left(x_{j}\right)=\left(g_{i}(\bar{\gamma}) \cdot x_{j}\right) ; \quad \forall i=1, \ldots, k ; j=1, \ldots, N .
$$

where $\Psi_{i}\left(x_{j}\right)$ are $k$ functional of the fields $x_{j}(t)$.

2) Then we define $\Phi_{i}(\gamma), k$ function of the curvilinear coordinates $\gamma$, by projecting the vacuum manifold $\mathscr{M}$, on the tangent space

$$
\Phi_{i}(\gamma)=\left(g_{i}(\bar{\gamma}) \cdot \tilde{x}(\gamma)\right) ; \quad \forall i=1, \ldots, k
$$

3) Eventually, due to the orthogonality conditions in Eq.s(3.3), for every lattice configuration $x_{j}(t)$ we can construct a system of $k$ equation for $k$ unknowns $\gamma_{i}$, by equating the functionals $\Psi_{i}\left(x_{j}\right)$ in

\footnotetext{
${ }^{1}$ For a complete discussion we refer to our upcoming paper [4].
} 
Eq.(3.8) with the functions $\Phi_{i}(\gamma)$ in Eq.(3.9)

$$
\left\{\begin{aligned}
\Psi_{1}\left(x_{j}\right)=\left(g_{1}(\bar{\gamma}) \cdot x_{j}\right) & =\left(g_{1}(\bar{\gamma}) \cdot \tilde{x}(\gamma)\right)=\Phi_{1}(\gamma) \\
& \ldots \\
\Psi_{k}\left(x_{j}\right)=\left(g_{k}(\bar{\gamma}) \cdot x_{j}\right) & =\left(g_{k}(\bar{\gamma}) \cdot \tilde{x}(\gamma)\right)=\Phi_{k}(\gamma) \quad ; \quad \forall j=1, \ldots, N .
\end{aligned}\right.
$$

Each system of equations generated by a given configuration $x_{j}$ has a set of curvilinear coordinates $\left\{\gamma_{i}\left[x_{j}\right]\right\}$ as a solution. By counting the occurence of each set of $\left\{\gamma_{i}\left[x_{j}\right]\right\}$ one can then reconstruct the probability distribution $P(\gamma)$ of the collective coordinates and hence the effective interaction $F(\gamma)$, given by

$$
F(\gamma)=-\log [P(\gamma)]
$$

The validity of this projection tecnique will now be tested on the simplest model presenting a non trivial vacuum structure.

\section{Double Well}

We now discuss the applications of our method to the quantum mechanical system described by a particle moving in a one-dimensional double well potential

$$
U(x)=m \alpha\left(x^{2}-\beta^{2}\right)^{2},
$$

where $m$ is the mass of the particle. We consider only paths with periodic boundary conditions

$$
x_{i}=x_{f}=-\beta \quad\left(\text { or equivalently: } x_{i}=x_{f}=+\beta\right) .
$$

The vacuum field manifold $\mathscr{M}$ for this model is the one generated by the superposition of $N$ instantons and $N$ antiinstantons. The collective coordinates are simply the Euclidean time coordinates of the pseudo-perticles $\gamma_{1}=t_{1}, \gamma_{2}=\bar{t}_{1}, \ldots, \gamma_{2 N-1}=t_{N}, \gamma_{2 N}=\bar{t}_{N}$ and the path integral may be written as

$$
Z[T ;-\beta,-\beta]=\int d t_{1}, \int d \bar{t}_{1} \ldots \int d t_{N} \int d \bar{t}_{N} e^{-\frac{1}{\hbar} F\left(t_{1}, \bar{t}_{1}, \ldots, t_{N}, \bar{t}_{N}\right)}
$$

If we consider the barrier to be infinitely high $\alpha \rightarrow \infty$ the instanton size vanishes and the both instanton and antiisnstanton will behave as non-interacting quasi-particles: this is the Dilute Instanton Gas Approximation (DIGA) regime and the multi-instanton effective interaction $F\left(t_{1}, . . \bar{t}_{N}\right)$ has an analitical expression. On the other hand, for a low barrier the DIGA fails to be a good approximation: in this regime, the vacuum fields behave as an interacting liquid and only single pair instanton-antiinstanton interactions are important

$$
F\left(\bar{t}_{1}, \ldots, \bar{t}_{N}\right) \simeq \sum_{i=1}^{N} F_{2}^{I A}\left(\bar{t}_{i}-t_{i}\right)+F_{2}^{A I}\left(t_{i+1}-\bar{t}_{i}\right),
$$

where $F_{2}^{I A}\left(F_{2}^{A I}\right)$ express the two-body instanton-antiinstanton (antiinstanton- instanton) correlations ${ }^{2}$. We then integrate all but a single instanton-antiinstanton pair, and rewrite the path integral as

$$
\left.Z[T ;-\beta,-\beta]=\frac{1}{2}\left(\int d t^{\prime} \int d t e^{-\frac{1}{\hbar} F_{2}^{I A}\left(t^{\prime}-t\right)}\right)+\int d t \int d t^{\prime} e^{-\frac{1}{\hbar} F_{2}^{A l}\left(t^{\prime}-t\right)}\right) .
$$

\footnotetext{
${ }^{2}$ Eq. (4.4) can be generalized to include higher-order (e.g. three-body, four-body, etc...) correlations.
} 
In order to extract the instanton-antiinstanton effective interaction $F_{2}^{I A}$ we then parametrize a generic configuration $x(t)$ using the sum ansatz for an instanton-antiiinstanton pair,

$$
\begin{aligned}
x(t) & =\tilde{x}^{I A}\left(t ; t_{1}, t_{2}\right)+y(t) \\
& =-\beta\left\{1-\tanh \left[\sqrt{2 \alpha} \beta\left(t-t_{1}\right)\right]+\tanh \left[\sqrt{2 \alpha} \beta\left(t-t_{2}\right)\right]\right\}+y(t)
\end{aligned}
$$

where $y(t)$ is a configuration of boundary conditions $y( \pm T / 2)=0$, and $t_{1}$ and $t_{2}$ are the coordinates of the two pseudoparticles, in the Euclidean time axis. We also define the variable $\xi=t_{2}-t_{1}$ which represents the "relative distance" between the instanton and antiinstanton: note that Eq.(4.5) implies

$$
F_{2}\left(t_{1}, t_{2}\right)=F_{2}\left(t_{2}-t_{1}\right) \equiv F_{2}(\xi)
$$

We now recall that the fluctuation field $y(t)$ have to fulfill the orthogonality conditions (3.3), which is enforced in a specific point $\gamma=\bar{\gamma}$ of the manifold $\mathscr{M}$. The vector basis of the tangent space of the manifold defined by our ansatz (4.6), for an arbitrary point $\gamma=\left(\bar{t}_{1}, \bar{t}_{2}\right)$ is

$$
\begin{aligned}
& g_{t_{1}}\left(t ; \bar{t}_{1}, \bar{t}_{2}\right)=\left.\quad \partial_{t_{1}} \tilde{x}_{S_{2}}\left(t ; t_{1}, t_{2}\right)\right|_{t_{1}=\bar{t}_{1}, t_{2}=\bar{t}_{2}}=-\sqrt{2 \alpha} \beta^{2} \operatorname{sech}^{2}\left[\sqrt{2 \alpha} \beta\left(t-\bar{t}_{1}\right)\right] \\
& g_{t_{2}}\left(t ; \bar{t}_{1}, \bar{t}_{2}\right)=\left.\quad \partial_{t_{2}} \tilde{x}_{S_{2}}\left(t ; t_{1}, t_{2}\right)\right|_{t_{1}=\bar{t}_{1}, t_{2}=\bar{t}_{2}}=\sqrt{2 \alpha} \beta^{2} \operatorname{sech}^{2}\left[\sqrt{2 \alpha} \beta\left(t-\bar{t}_{2}\right)\right]
\end{aligned}
$$

Now that the fields $\tilde{x}$ and $y$ have been defined, we are ready to determine the effective interaction $F_{2}^{I A}(\xi)$ using the projection tecnique presented in Section 3. In order to show that our method reproduces the correct result, we first consider the two instanton-antiinstanton interaction in the DIGA regime, which has analytical solution

$$
F_{2, D I G A}^{I A}(\xi)=(\kappa T-1) \log (T-\xi)+\text { const, }
$$

where $k$ is a constant which can be interpreted as the instanton density. By sampling and projecting equilibrium configurations generated with the probability distribution $e^{-F_{2, D I G A}^{I A}}$ we obtain the results in Fig.(2): the dashed line is the expected theoretical result. The DIGA approximation holds only at large instanton-antiinstanton distance, i.e. for $\xi$ much larger than the instanton size - which is 0.26 , in these units-. In this regime our projection tecnique reproduces the correct result; in fact, a linear fit of the data for $\xi>1$ yields a slope of $0.32 \pm 1$, in excellent agreement with the exact theoretical result, which is 0.31 . We now consider quantum fluctuations and reconstruct the full non-perturbative effective interaction for high and low barrier: Fig. (3) shows the results of such a non-perturbative calculation for a well with $\alpha=1$ (low barrier) and $\alpha=7$ (high barrier). As one can see, at small $\xi$-i.e. when instanton and antiinstanton overlap- quantum interactions induce an effective repulsion, resulting in a minimum of the effective interaction at finite postitive $\xi$ : this effect is enhanced in the lower barrier. In such a regime, the vacuum behaves like a onedimensional liquid, rather than as an ideal gas; we note that this is precisely the physical picture underlying the instanton liquid model of the QCD vacuum. For higher values of $\xi$ the effective interaction starts to raise and eventually reaches the dilute gas limit. 


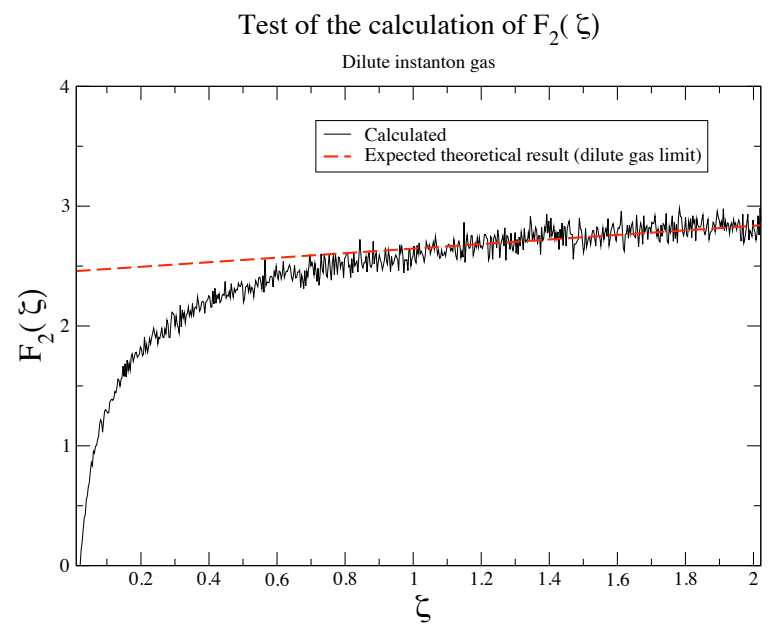

Figure 2: Non-perturbative calculation of the effective interaction $F_{2}(\xi)$ for a dilute instanton gas with $\alpha=7, m=1, \beta=1$ in a volume $T=200$.
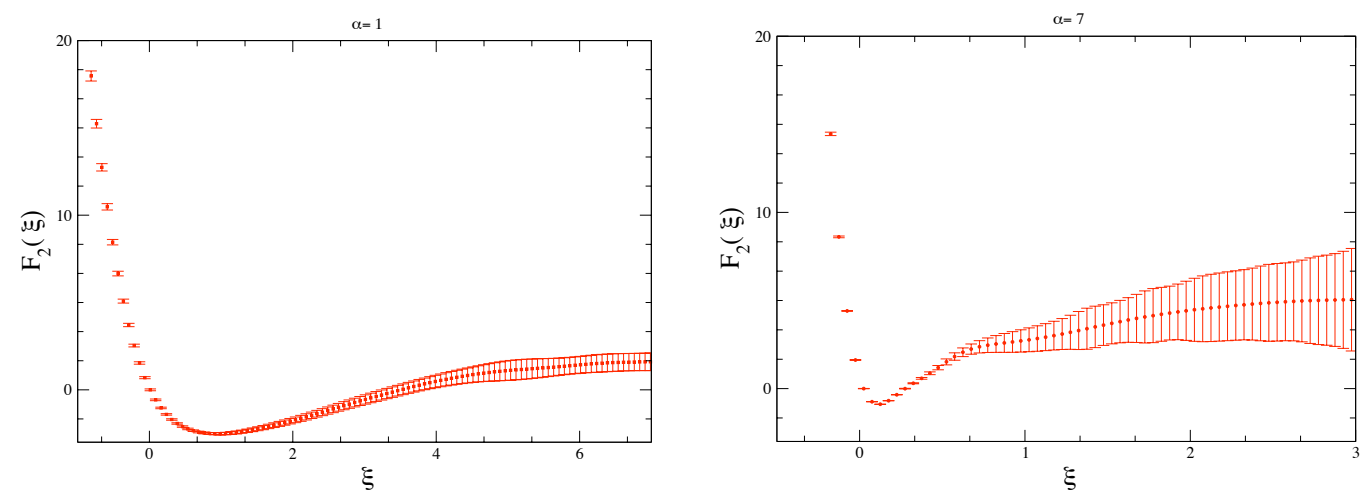

Figure 3: Non-perturbative calculation of the effective interaction $F(\xi)$ for $\alpha=1$ (left panel) and $\alpha=7$.

\section{Conclusions}

We presented a novel approach which allows to express quantum mechanical path integrals, in terms of few ordinary integrals over a set of low-energy variables, which parametrize the manifold of the relevant vacuum field configurations. The method consists in reconstructing numerically the effective theory for a given family of vacuum fields, using lattice simulations. In this work we discussed the results for the instanton-antiinstanton effective interaction in the one-dimensional double well potential. The extension of the present formalism to instantons in QCD is in progress and will be presented in our upcoming papers. 


\section{References}

[1] D.Diakonov, Chiral Symmetry breaking by instantons, Lectures given at the "Enrico Fermi" school in Physics, Varenna, June 25-27 1995, hep-ph/9602375. P.Faccioli and T.A.DeGrand, Phys.Rev.Lett.91 (2003) 182001

[2] M.Cristoforetti, P.Faccioli,M.Traini, Phys.Rev.D75 054024 (2007).M.Cristoforetti, P.Faccioli,M.Traini and J.W.Negele, Phys.Rev.D75 034008 (2007). M.Cristoforetti, P.Faccioli, Phys.Rev.C69 (2004)065211. M.Cristoforetti, P.Faccioli, E.V.Shuryak e M.Traini, Phys.Rev.D70 (2004) 054016

[3] T. Schaefer and E. Shuryak, Rev. Mod. Phys. 70, 323 (1998).

[4] R.Millo, L.Scorzato and P.Faccioli, arXiv:0911.3021 (2009). 